\title{
Defaulting patterns in a provincial leprosy control programme in Northern Mozambique
}

\author{
STEPHEN GRIFFITHS \& NATASHA READY \\ Direcção Provincial da Saúde, Cidade de Pemba, Cabo Delgado, \\ Moçambique
}

\author{
Accepted for publication 1 May 2001
}

\begin{abstract}
Summary Cohort-based multidrug therapy (MDT) completion rates are used to assess adherence to MDT. However this measure gives no information about when during the treatment period defaulting occurs. Two districts in Cabo Delgado province in Northern Mozambique were selected for evaluation of multibacillary patient defaulter data between 1993 and 1997 to examine when patients default during the treatment period. In all, $548(59 \cdot 2 \%)$ of $926 \mathrm{MB}$ patients completed treatment and $378(40.8 \%)$ defaulted between 1993 to 1997 . The percentage of defaulters fell steadily from $59.8 \%$ in 1993 to $23.2 \%$ in 1997 . Of the 378 defaulters $57.7 \%$ defaulted treatment within 6 months and $83.1 \%$ within 1 year of starting treatment. It was observed that patients tend to default early rather than late in the treatment period and that this pattern is maintained over time despite a fall in defaulter rates. Patients established early into a treatment routine were more likely to complete treatment. A comprehensive effort to improve and maintain leprosy control services will probably influence adherence more than any single, specific strategy. Shortening MDT treatment from 2 years to 1 year is unlikely to affect the defaulter rate.
\end{abstract}

\section{Introduction}

In December 1992, following the cessation of hostilities in Mozambique, the Provincial Tuberculosis/Leprosy Control Programme (Estratégia de Luta Anti-Tuberculose/Estratégia de Luta Anti-Lepra Provincial) of the Ministry of Health was restarted in Cabo Delgado Province, Northern Mozambique with technical, logistical and financial support from The Leprosy Mission International. The Provincial Tuberculosis/Leprosy Control Programme aimed to serve a population of $1,284,480$ people in an area of 83,000 square kilometres. ${ }^{1}$ Cabo Delgado remains one of the poorest provinces in the country, with lower literacy rates than the national average ( $18 \%$ adult literacy) and unreliable communication and supply systems. There was no telephone link from provincial to national capital. There was no direct road connection as bridges across the Zambezi had been destroyed. There was no rail

Correspondence to: S. D. Griffiths, PO Box 1490, Phnom Penh, Cambodia (e-mail: GriffithsStephen@omf.net) 
connection, so re-supply of drugs could take months by ship. Sometimes this meant that drugs (including MDT) would run out altogether and health institutions were without some drugs for several months.

The Provincial Medical Directorate had 30\% of staffing levels at health institutions recommended by the Ministry of Health's own guidelines. There was poor geographical coverage by the health system with populations of up to 30,000 people with no health facility within $80 \mathrm{~km}$. Poor roads, minefields and banditry made travel hazardous. Only eight of 17 district health directorates had even one vehicle in 1992 and fuel was often not available. There was inadequate physical infrastructure at peripheral, district and provincial levels. Around one-third of health institutions had been destroyed during the civil war and so a health centre sometimes consisted of a table under a tree. Of those that were left most had not been maintained for more than 20 years or had lost doors, windows and roof to theft. Only three district health centres had electricity. Health centres up to and including some district heath centres consisted of pole and dagga huts with a very low level of lighting, comfort and hygiene. Drugs, equipment and patient files were often damaged by rain, termites or rats. Even pens were in short supply and leprosy notifications written in charcoal were received from some districts.

In December 1992 the leprosy control component of the combined Provincial Tuberculosis/Leprosy Control Programme was weak. The registered leprosy prevalence rate for the province was 33 per 10,000 population. Only $14 \%$ of the 3883 patients registered for treatment were on MDT, the remainder theoretically on dapsone monotherapy. There were 11 drug delivery points for the entire province. Supervision by provincial staff was infrequent only four districts were visited once each during 1992 . The case detection rate was $2.2 / 10,000$ population. For those on MDT, the provincial $1992 \mathrm{MB}$ cohort had a treatment completion rate of $20 \cdot 1 \%$ (44 of 222 patients completed treatment).

Improvement of the leprosy control component of the Provincial Tuberculosis/Leprosy Control Programme began in 1993. Six District Tuberculosis/Leprosy Control Programme (DTBLCO) supervisors were selected each year and sent to a 6-week national tuberculosis/ leprosy (TB/L) training course. They were then based one in each of 17 district administrative centres. Regular supervisory visiting (at least once in every 6 months for every district) was initiated and a standardized supervision report form was in use by late 1996 together with regular in-service training. Reserve MDT stocks were created at provincial and district levels.

In 1995,57 peripheral health post staff and 120 village health workers were trained to assist in TB/L control. This allowed the opening of a further 123 treatment points beyond the 17 district health centres. From 1995 DTBLCO supervisors were supplied with motorbikes, which allowed regular visiting, technical support and logistical re-supply of peripheral health centres. Blister calendar packs of MDT were introduced to simplify the handling and distribution of drugs. Funding was made available for each DTBLCO supervisor's activities, based on an annual work plan drawn up by each district. All interventions were concentrated on improving the quality of service offered.

The provincial leprosy service was adjudged to have improved during the period under study; during December 1997, the percentage of patients on MDT had risen from $14 \%$ to $100 \%$, the leprosy case detection rate had risen from $2.2 / 10,000$ to $4.7 / 10,000$ and the provincial leprosy prevalence rate had fallen from $33 / 10,000$ to $10 / 10,000$ through review and removal of patients who no longer required chemotherapy.

The treatment completion rate for the 1996 multibacillary (MB) cohort at provincial level was assessed in September 1999 (by which time it was possible to determine an entire cohort 
outcome) and had risen from $20 \cdot 1 \%$ to $73.7 \%$ (590 of 801 patients completed treatment). All districts were supervised, restocked with drugs and notified statistics at regular intervals.

This study had two main objectives: (i) to discover at what point MB patients default during MDT treatment and (ii) if improvements in the leprosy control programme had an effect on the number of doses patients received before defaulting.

\section{Materials and methods}

Chiúre and Namuno districts were selected because they had stable leprosy control programmes between 1993 and 1997. Relatively reliable registration and patient records were available. Chiúre's population in 1997 was 189,100 inhabitants and Namuno's was 137,275 inhabitants. ${ }^{1}$ Both districts are land-locked with the vast majority of the population involved in the subsistence farming of cassava and maize, and growing cashew nuts and cotton for cash income.

Chiúre and Namuno districts were visited in October and November 1999 and all records available were reviewed with the DTBLCO supervisors. All patients classified as MB (i.e. more than five skin lesions) who entered the leprosy control programme in Chiúre and Namuno districts between January 1993 and December 1997 were taken. Treatment registers were examined and the number of supervised monthly doses taken before defaulting recorded for each MB defaulter. An MB defaulter was defined as a patient diagnosed with leprosy and classified as being multibacillary who had failed to take 24 supervised monthly doses in a total period of 36 months. ${ }^{2}$ This definition remained constant from 1993 to 1997. From mid1999 onwards, the National Tuberculosis/Leprosy Control Programme reduced the treatment period for MB cases from 24 supervised monthly doses to 12 supervised monthly doses so the last cohort studied was the 1997 MB cohort. All patients in these two districts had a defined outcome before the change in policy took effect despite the fact that the cohort was only due to be evaluated in January 2000.

To simplify the presentation of the data the number of doses taken were divided into eight 3-month periods. Each defaulting patient was assigned to a period depending on the number of supervised monthly doses he/she had received before defaulting.

\section{Results}

A total of $930 \mathrm{MB}$ patient records were available. Four patients either died or were transferred before completing treatment and were excluded from the study. The DTBLCO supervisor's records showed that $548(59 \cdot 2 \%)$ of $926 \mathrm{MB}$ patients completed treatment and $378(40 \cdot 8 \%)$ defaulted during the period 1993 to 1997.

Table 1 shows the numbers of patients in each district defaulting over the time span of the study period against the total number of patients entering the programme. This shows a steady fall in the percentage of defaulters between 1993 and 1997.

Table 2 shows the distribution of the numbers of patients by doses taken before defaulting in each dosage category. Most patients defaulted early in the treatment period rather than later. This pattern was sustained year by year throughout the period under review. Of 378 defaulters $57.7 \%$ defaulted treatment within 6 months and $83.1 \%$ within 1 year of starting treatment. Figure 1 shows this information. 


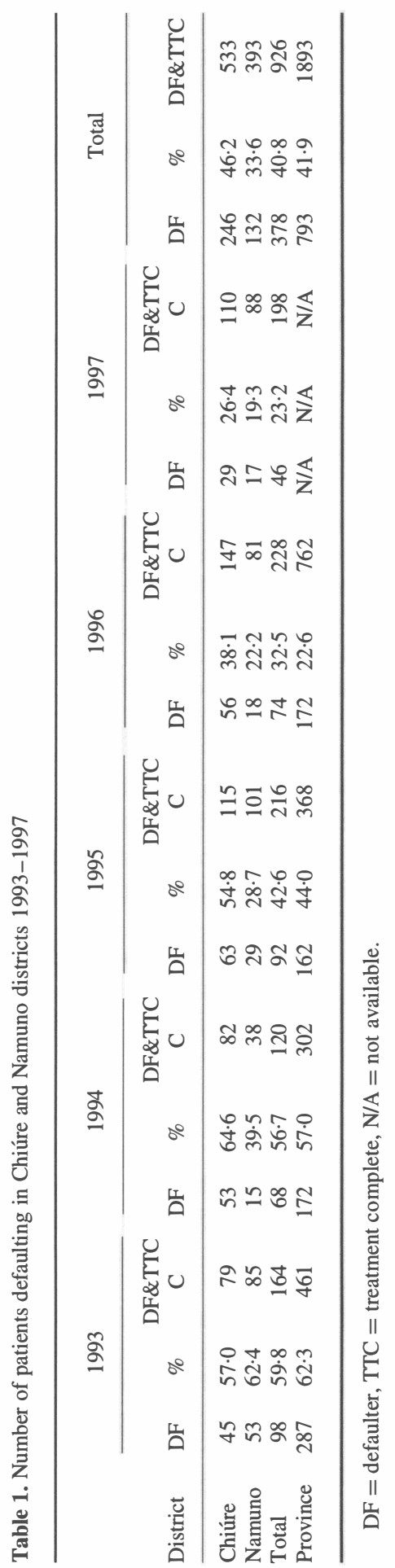


Table 2. Distribution of number of patients by doses taken before defaulting in Chiúre and Namuno districts 1993-1997

\begin{tabular}{lrrrrrrr}
\hline Doses & 1993 & 1994 & 1995 & 1996 & 1997 & Total defaulters & $\%$ of total \\
\hline $1-3$ & 29 & 24 & 26 & 27 & 12 & 118 & $31 \cdot 2$ \\
$4-6$ & 25 & 16 & 14 & 27 & 18 & 100 & $26 \cdot 5$ \\
$7-9$ & 14 & 5 & 11 & 13 & 12 & 55 & $14 \cdot 6$ \\
$10-12$ & 15 & 4 & 13 & 6 & 3 & 41 & $7 \cdot 8$ \\
$13-15$ & 9 & 3 & 13 & 1 & 1 & 20 & $5 \cdot 1$ \\
$16-18$ & 3 & 6 & 11 & 0 & 0 & 0 & $4 \cdot 5$ \\
$19-21$ & 3 & 10 & 4 & 0 & 0 & 378 & 0 \\
$22-23$ & 0 & 0 & 0 & 0 & 0 & & \\
Total & 98 & 68 & 92 & 74 & 46 & & \\
\hline
\end{tabular}

\section{Discussion}

We thought that the distribution of the number of patients defaulting plotted against the number of supervised monthly doses taken before defaulting would have a Gaussian distribution. Few patients would default early, few would default late but the bulk of patients would default in the middle of the treatment period. We predicted that improvements in the leprosy control programme would result in more patients taking mores doses of MDT before defaulting. Unfortunately, it was not possible to compare defaulter rates in Cabo Delgado province with other provinces as they simply reported the annual total number of defaulters and did not break these figures down by classification or by entry cohort. ${ }^{2}$

We believed the package of interventions undertaken in this leprosy control programme should have caused a consistent reduction in the number of patients defaulting annually

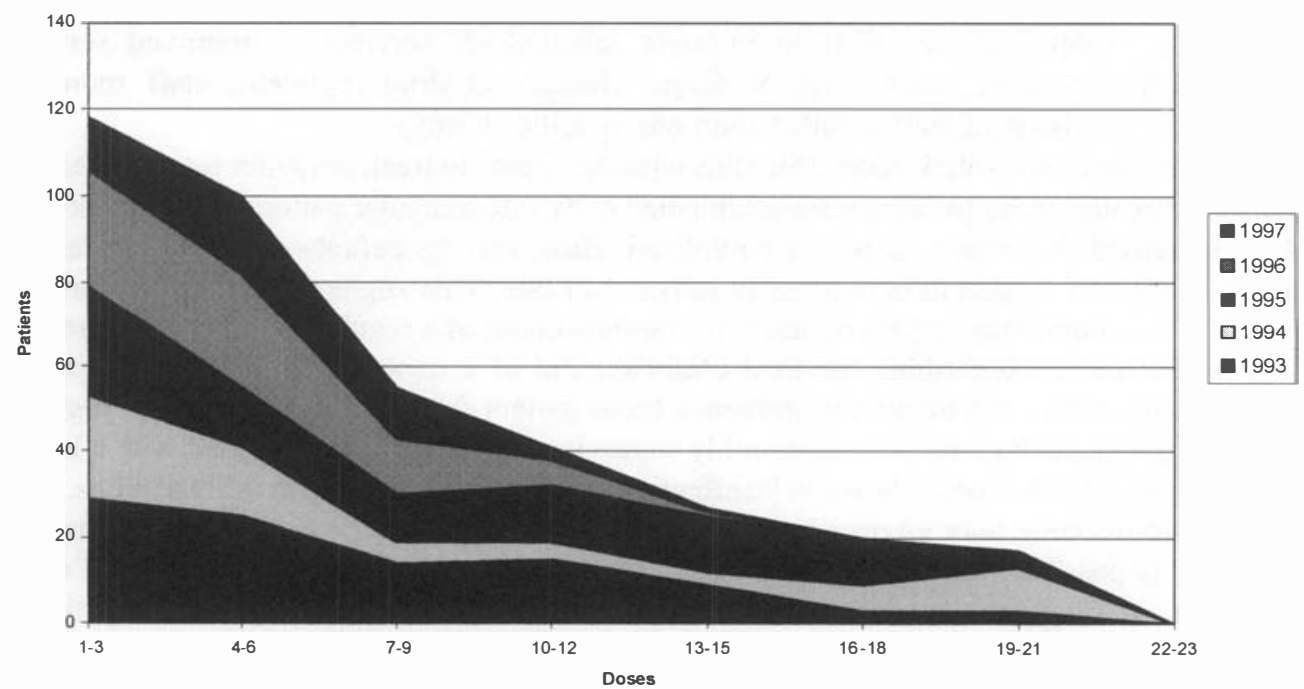

Figure 1. Distribution of number of patients by doses taken before defaulting in Namuno and Chiure between 1993 and 1997. 
despite a rise in the detection rate increasing the new MB caseload. However, instead of a gradual increase in the number of supervised monthly doses taken before defaulting, resulting in a progressive rise in patients who actually completed their treatment period, it would seem from our data that an improved service resulted in a reduced proportion of defaulters but did not change the pattern of defaulting from the programme. Once patients were established in a treatment routine, they were likely to complete. If a good treatment routine was not established within the first few visits patients were much more likely to default. A study in $\mathrm{Nepal}^{3}$ reached the same conclusion.

'To complete treatment is usually an independent choice of patients' ${ }^{4}$ But for a successful treatment outcome, there needs to be input from both health service provider and client seeking care. In Mozambique at the outset of the study period, the ability to complete treatment was probably more often determined by the low quality of the leprosy service rather than patient choice. For example, after 4 months without drugs in one district in 1993, it is hardly surprising that all leprosy patients there defaulted. A few contacts with an inadequate leprosy service was probably enough to discourage most patients from returning.

On questioning DTBLCO supervisors about the initial high defaulter rate in Cabo Delgado, the explanation consistently given was what might be termed the 'bad patient' hypothesis. The patients were variously described as lazy, ignorant and not interested in their own health, despite the fact that all patients had come seeking help in the first place as case finding was entirely passive. However, as staff were trained, were motivated by regular supervision and meetings and were re-supplied with drugs, they saw the defaulter rate fall despite the fact that there was no attempt at mass health education or improving community awareness of leprosy and duration of leprosy treatment. We observed that over time the 'bad patient' hypothesis was less frequently offered by staff as an explanation for defaulting.

Directly observed treatment for tuberculosis has been associated with substantial improvements in rates of adherence in some countries. ${ }^{4}$ However, a study in Pakistan showed no advantage of directly observed treatment over self-administered or family observed treatment. ${ }^{5}$ However, both papers showed improvement in adherence rates as a result of a comprehensive effort to improve tuberculosis services - improved service accessibility, increased availability of drugs, changes in drug regimens, staff training, increased supervision of staff - rather than one specific strategy.

With trained, motivated, accessible staff with the means to treat, defaulter rates decreased probably because more patients were established early into a regular pattern of treatment and so an increased proportion each year completed. However, the defaulter rate did not fall to zero and patients tended to default early rather than late. This suggests that a good quality control programme may impact on the early establishment of a regular treatment routine but then patient factors determine the final establishment of a treatment routine. The leprosy service may or may not be able to influence these patient factors. Heynders et al. ${ }^{3}$ suggest targeting patients who miss several monthly supervised doses early in treatment with a letter or home visit. This has been shown to be effective in reducing defaulting in other settings. But in a human-resource poor environment, where travel is time-consuming and patients widespread, it is possible that time spent away from the health centre by leprosy control staff following up irregular attenders will adversely affect the treatment outcome for patients coming to the health centre. Perhaps peer help (preferably volunteer) as animators would be more useful, particularly in the collective village setting. Clear, structured health education guidelines for health staff together with a take-home leaflet for patients, appropriate to a population with low literacy skills, would be two further strategies to examine. Due to the 
complex nature of non-adherence, probably a combination of strategies will be found to work best.

The recent introduction of a 12-dose fixed duration regime for MB patients in Cabo Delgado province will probably have only a minor effect on improving the defaulter rate further. Improving and maintaining the quality of a leprosy control service is more likely to keep defaulter rates low than the reduction of the length of treatment from 2 years to 1 year. Since the default rate has changed but the default pattern has remained constant, further research would be justified in looking at factors influencing why those patients that default continue to do so early despite an improved leprosy control service.

\section{References}

1 Anonymous. Instituto Nacional de Estatística. Estimatívas e Projecções da População de Moçambique 19902000. Maputo, Moçambique, Maio 1998.

2 Compostella L. Ministério da Saúde de Moçambique. Manual de Lepra. Maputo, Moçambique, 1991.

${ }^{3}$ Heynders ML, Meijs JJ, Anderson AM. Towards an understanding of non-compliance. An assessment of risk factors for defaulting from leprosy treatment. Lepr Rev, 2000; 71: 369-376.

${ }^{4}$ Gamer P, Volmink J. Systematic review of randomised controlled trials of strategies to promote adherence to tuberculosis treatment. BMJ, 1997; 315: 1403-1406.

5 Walley JD, Khan MA, Newell JN, Khan MH. Effectiveness of the direct observation component of DOTS for tuberculosis: a randomised controlled trial in Pakistan. Lancet, 2001; 357: 664-669. 